\title{
Imprint cytology versus frozen section analysis for intraoperative assessment of sentinel lymph node in breast cancer
}

This article was published in the following Dove Press journal:

Breast Cancer - Targets and Therapy

5 May 2017

Number of times this article has been viewed

\section{Thalia Petropoulou' \\ Antonia Kapoula ${ }^{2}$ \\ Aikaterini Mastoraki ${ }^{3}$ \\ Aikaterini Politi \\ Eleni Spanidou-Karvouni ${ }^{2}$ \\ loannis Psychogios' \\ loannis Vassiliou' \\ Nikolaos Arkadopoulos ${ }^{3}$}

'2nd Department of Surgery, 2Department of Pathology, Aretaieion University Hospital, ${ }^{3}$ th Department of Surgery, Athens University Medical School, Attikon University Hospital,

Chaidari, Athens, Greece
Correspondence: Aikaterini Mastoraki 29 Irodotou Street, I5 22 Maroussi,

Athens, Greece

Tel +306932577710

Fax +302105326412

Email dr_kamast@yahoo.gr
Introduction: Sentinel lymph node (SLN) biopsy is the gold standard for surgical staging of the axilla in breast cancer (BC). Frozen section (FS) remains the most popular means of intraoperative SLN diagnosis. Imprint cytology (IC) has also been suggested as a less expensive and equally accurate alternative to FS. The aim of our study was to perform a direct comparison between IC and FS on the same SLNs of BC cases operated in a single center by the same surgical team. Materials and methods: Into this prospective study we enrolled 60 consecutive patients with histologically proven T1-T3 BC and clinically negative axilla. Sentinel nodes were detected using a standard protocol. The SLN(s) was always assessed by IC as well as FS analysis and immunohistochemistry. Nevertheless, all intraoperative decisions were based on FS analysis.

Results: During the study period 60 patients with invasive BC were registered, with 80 SLNs harvested. Mean number of SLN(s) identified for each patient was 1.33. The sensitivity and specificity were $90 \%$ and $100 \%$, respectively, for IC, and $80 \%$ and $100 \%$ for FS. Relevant positive/negative predictive values were $100 \% / 98 \%$ for IC and $100 \% / 96.15 \%$, respectively, for FS. Overall accuracy was $98 \%$ for IC and $97 \%$ for FS. Therefore, statistically significant difference between the two methods in the detection of positive nodes was not elucidated $(p=1.000)$. Conclusions: IC appeared to be marginally more sensitive than FS in detecting SLN metastatic activity. Overall accuracy was $98.75 \%$. With regard to the primary lesion characteristics, we conclude that initial lesion size and lymphovascular invasion play a pivotal role in metastatic involvement of the SLN with the dimensions of metastasis bearing no correlation with tumor size. Therefore, IC appears to be a sensitive and accurate method for the intraoperative assessment of SLN in BC patients, but further studies are required to confirm this interesting data. Keywords: breast cancer, sentinel lymph node biopsy, frozen section, imprint cytology

\section{Introduction}

Sentinel lymph node (SLN) biopsy is the gold standard for surgical staging of the axilla in breast cancer (BC). ${ }^{1,2}$ Intraoperative pathologic assessment of SLNs is desirable, because it may spare the patient a second operation if a preoperative decision has been made to proceed with completion axillary lymph node dissection (ALND) in case of positive SLN(s). ${ }^{3-6}$ Frozen section (FS) remains the most popular means of intraoperative SLN diagnosis in many centers although the overall rate of intraoperative SLN pathologic assessment appears to be declining, probably as an aftermath of the American College of Surgeons Oncology Group Z0011 Trial. ${ }^{7}$ The use of FS is accompanied with many drawbacks such as considerable waiting time, tissue loss, and cost. Furthermore, false-negative rates are always a concern. ${ }^{8}$ Intraoperative imprint cytology (IC) has also been suggested as a less expensive and equally accurate alternative to FS; this 
technique can also be performed on lymph nodes replaced by fat. ${ }^{9,10}$ Nevertheless, a higher probability of false-negative results has been recorded and the presence of micrometastasis cannot be excluded. ${ }^{11-13}$ Sensitivity also depends on the histologic type of the tumor, with lower rates of detection for lobular carcinoma (LC) in comparison with the more common ductal type. ${ }^{14,15}$ Additional immunohistochemical staining may aid in the detection of micrometastases even in LC and contributes to the increase of IC sensitivity. ${ }^{16}$ The aim of our study is to perform a direct, head-to head comparison between IC and FS on the SLNs of BC cases operated in a single center by the same surgical team in order to determine which method should be adopted as our standard method of intraoperative SLN evaluation in our academic unit.

\section{Materials and methods \\ Patient selection criteria}

Into this prospective study we enrolled 60 consecutive patients with histologically proven T1-T3 BC and clinically negative axilla who were admitted by a single surgical team to the Breast Unit of our university hospital from January 01, 2011, to December 31, 2013. The Institutional Review Board of Aretaieion University Hospital approved the study according to National and European legal standards. Furthermore, written informed consent was obtained by all the participants. In each patient SLN biopsy prior to ALND was implemented. Exclusion criteria were T4 tumors and/or clinically positive axilla, pregnancy, or lactation.

\section{SLN biopsy protocol}

Sentinel nodes were detected using a standard protocol of localization using radiopharmaceutical and blue dye injection intraoperatively, as previously described. ${ }^{17}$ Briefly the technique includes subcutaneous administration of 20-40 $\mathrm{MBq}$ of Nanocoll into the periareolar region within 2 hours prior to surgery. After induction of anesthesia an additional $2 \mathrm{~mL}$ of patent blue was injected in the periareolar area. Subsequently, the axilla was systematically scanned visually with a handheld gamma probe providing three-dimensional orientation about the precise location of the SLN. Preoperative lymphoscintigraphy was performed in $60 \%$ of cases.

Surgical criteria for SLN(s) include the identification of all "hot" and blue nodes as previously described (Figure 1). ${ }^{18}$ The SLN(s) was always assessed by IC as well as FS analysis and IHC. During SLN assessment in the laboratory the patient was submitted to the scheduled definitive operation for BC such as wide local excision or mastectomy with or without reconstruction. In case of macrometastatic disease in the

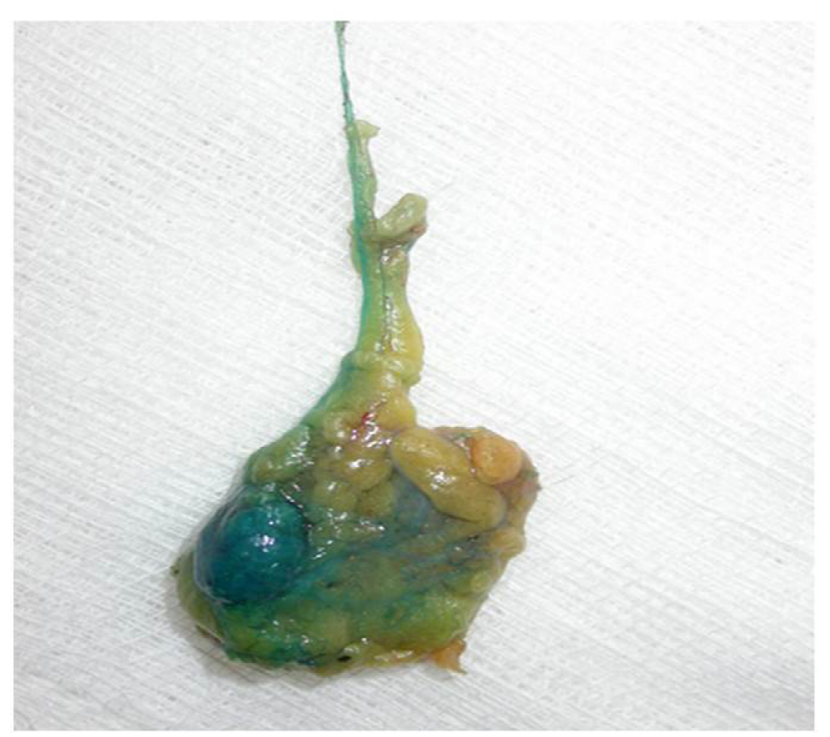

Figure I Identification of "hot" and blue nodes in a surgical specimen of sentinel lymph node biopsy.

SLN(s) the patient underwent the standard ALND. According to the design of the survey all intraoperative decisions were based on FS analysis, which has been the standard of care in our surgical department for many years.

\section{Intraoperative IC}

The SLN(s) were received fresh in the department of pathology. SLN(s) greater than $5 \mathrm{~mm}$ in size were sliced to sections of 2-mm thickness, whereas SLN(s) less than $5 \mathrm{~mm}$ were bisected. Two slides of touch imprints were provided from each section of the SLN and were air-dried. The first one was stained with the Wright-Giemsa method (Hemacolor rapid staining, Merck KGaA, Darmstadt, Germany) and assessed immediately by an experienced cytopathologist. The other one was fixed in cold acetone for $30 \mathrm{sec}$, allowed to dry, and rinsed in phosphate buffer saline.

\section{Intraoperative FS and IHC}

In all cases two sections were cut in the cryostat and subsequently stained with hematoxylin and eosin (H\&E) for FS analysis and assessed by another dedicated cytopathologist. The rest of the tissue as well as the FS blocks were embedded in paraffin. From each block, three slides were obtained and additional two or three sections were stained with $\mathrm{H} \& \mathrm{E}$. The results of both IC and FS examination of the SLN(s) were communicated to the surgical team as soon as they were available, during the ongoing surgical procedure. If the cytomorphology was not diagnostic a rapid immunohistochemical analysis was performed in the second slide using the Dako REAL Envision detection system (DAKO, 
Glostrup, Denmark) and cytokeratin AE1/AE3 as the primary antibody. After completion of the intraoperative assessment, the lymph node sections were fixed in $10 \%$ neutral buffered formalin overnight, followed by paraffin embedding of the entire specimen and routine postoperative histopathological evaluation.

\section{Statistical analysis}

Variables were investigated for normality of distributions using the Kolmogorov-Smirnov test. Comparisons of numeric data between the two groups were performed with the independent unpaired $t$-test with regard to mean size of the primary tumor or metastatic foci. Mann-Whitney statistical analysis was employed in case of violation of normality. Comparisons of categorical data were carried out by the method of chi-square or Fisher's exact test, as appropriate. Pairwise multiple comparisons were established with McNemar's test. All analyses were executed using SPSS version 13.0 , and $p<0.05$ was indicated as the statistically significant level.

\section{Results}

During the study period, 60 patients with invasive BC were registered, with $80 \mathrm{SLNs}$ harvested. Average age was 61.3 years (range 31-82). Mean number of SLN(s) identified for each patient was 1.33. Mean tumor size was significantly larger $(2.34 \pm 1.53 \mathrm{~cm})$ in patients with axillary metastatic disease in comparison with SLN-negative women $(1.58 \pm 0.68 \mathrm{~cm} ; p=0.016)$. Meticulous postoperative histological examination revealed 10 positive and 50 negative cases (Figure 1). False-positive results were not reported. Among SLN-positive patients we detected six ductal adenocarcinomas, one LC, and three cases of mixed ductal/lobular type with seven macrometastases and two micrometastases elucidated. One case of isolated tumor cells in an SLN was detected.

The sensitivity and specificity was $90 \%$ and $100 \%$, respectively, for IC and $80 \%$ and $100 \%$ for FS. Relevant positive/negative predictive values were $100 \% / 98 \%$ for IC and $100 \% / 96.15 \%$, respectively, for FS. Overall accuracy was $98 \%$ for IC and $97 \%$ for FS. Therefore, we found no statistically significant difference between the two methods in the detection of positive nodes $(p=1.000)$. Furthermore, comparison of FS analysis with the combination of IC and IHC provided similar evidence $(p=0.5)$. Additionally, the overall accuracy in detecting macrometastases was $100 \%$ for IC and $97.5 \%$ for FS with relevant sensitivity rates $100 \%$ and $89 \%$, respectively. False-positive results were absent. Moreover, a statistically significant correlation has been suggested between FS and IHC $(p<0.001)$, FS and IC $(p<0.001)$ and IHC and the combined $\mathrm{IC}+\mathrm{IHC}$ technique $(p<0.001)$ with regard to the time required to the completion of the histological examination. Results are summarized in Table 1.

Study participants included 10 premenopausal and 50 postmenopausal women. No statistically significant difference was found between the ratio of premenopausal and postmenopausal state in women with positive SLN $(20 \%$ versus $16 \%, p=0.668)$. Furthermore, 42 patients were defined as T1 tumors, 16 women were characterized as T2 lesions, and two patients appeared with T3/T4 tumors. Additional statistical analysis identified that $\mathrm{T}$ stage was correlated significantly to metastatic involvement of SLN ( $p=0.016)$. In addition, the size of metastases on the $\operatorname{SLN}(s)$ bore no correlation with the $\mathrm{T}$ stage of the primary tumor $(p=0.977)$. On the other hand, there is a trend for positive correlation between the percentage of positive SLNs and the extent of metastatic disease $\left(r^{2}=0.362, p=0.086\right)$ as well as the tumor

Table I Tumors' characteristics in invasive breast cancer patients submitted to sentinel lymph node biopsy

\begin{tabular}{ll}
\hline Patients & $\mathbf{N}=\mathbf{6 0}$ \\
\hline SLNs & 80 \\
SLN/Patient & 1.33 \\
Mean age & 61.3 \\
Mean tumor size in SLN (+) patients & $2.34 \pm 1.53 \mathrm{~cm}$ \\
Mean tumor size in SLN $(-)$ patients & $1.58 \pm 0.68 \mathrm{~cm}$ \\
Positive cases & 10 \\
Negative cases & 50 \\
\hline
\end{tabular}

Abbreviation: SLN, sentinel lymph node.

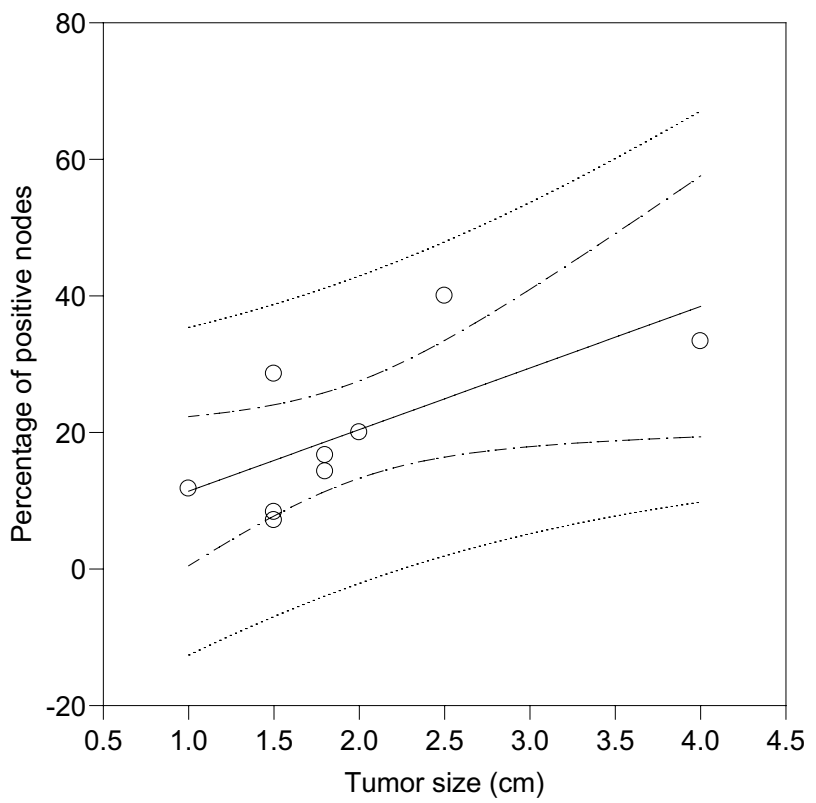

Figure 2 Correlation between the percentage of positive sentinel lymph nodes and tumor size in 60 breast cancer patients. 
size $\left(r^{2}=0.464, p=0.043\right)$ (Figure 2$)$. Indications of extranodal extension of nodal metastasis were not apparent.

Among the 60 patients of this study, estrogen receptors (ER) positivity was apparent in 53 cases and progesterone receptors (PgRs) positivity in 43 samples. Moreover, all patients with positive SLN ( $\mathrm{n}=10)$ were ER positive. Nevertheless, the percentage of positive ERs and PgRs was not significantly different between women with axillary involvement versus those with negative SLN biopsy ( $p=0.589$ and $p=0.709$, respectively).

\section{Discussion}

Imprint or touch-preparation cytology of SLNs has been established in the literature as an effective modality with high specificity and a wide range of sensitivity rates. In accordance with a recent meta-analysis including 31 methodologically acceptable surveys, the pooled sensitivity of IC was $63 \%$ with specificity levels as high as $99 \%$. Associated pooled sensitivity for macrometastases detection was $81 \%$ and that for micrometastases determination only $22 \%$. Mean or median primary tumor size, the prevalence of metastases, and the proportion of micrometastatic evidence proved significant determinants of the outcome in single-variable meta-regression analysis. ${ }^{5}$ Moreover, the proportion of micrometastases appeared the only significant risk factor in multivariable examination. Finally, frozen sectioning had better sensitivity compared to IC in three among four direct comparative evaluations. ${ }^{19}$

IC is a simple, rapid, and cost-effective technique for axillary assessment, which also preserves the SLN for further histological examination. Interpretation by an experienced cytopathologist is always required. ${ }^{20,21}$ Therefore, IC is operator dependent as reflected in the wide range of success in the published reports. On the other hand, FS has been described as the method of choice for intraoperative evaluation of the nodal status of the axilla for decades. Nevertheless, despite its reliability, FS analysis is a time-consuming technique as well as labor intensive and expensive. Our results depicting sensitivity and specificity rates of $90 \%$ and $100 \%$, respectively, are comparable with previously published data for FS and IC. . $2-25^{2}$

When IC is combined with IHC, the sensitivity and specificity are $100 \%$. Even though IHC is a very sensitive and accurate method, additional cost and time required exclude it as a routine diagnostic procedure. Nevertheless, in case of questionable diagnosis or morphologic uncertainty with regard to potential detection of atypical cells implying the presence of micrometastases or istiocytes, an immunohistochemical examination is often warranted. ${ }^{26}$ Increasing the number of serial sections has been suggested to improve sensitivity. Recently, intraoperative ultrarapid IHC has been investigated for its feasibility, validity, and effectiveness in comparison with FS. Ultrarapid cytokeratin IHC significantly enhanced intraoperative detection of metastasis in SLNs without increased time for assessment (Table 2). This technique is currently not widely available and requires specialized expertise. Other experimental techniques such as intraoperative multiparameter flow cytometry and real-time polymerase chain reaction for cytokeratins in SLNs have also yielded promising results..$^{27,28}$

LC presents a low-grade cytomorphology similar to lymphoid cellular structure. Therefore, acute identification of axillary metastatic disease on both IC and definite postoperative histology remains problematic. Additional

Table 2 Comparison of imprint cytology, frozen sectioning, and immunohistochemistry analysis in invasive breast cancer patients submitted to sentinel lymph node biopsy

\begin{tabular}{|c|c|c|c|c|c|c|c|}
\hline & \multirow{2}{*}{$\begin{array}{l}\text { Number of } \\
\text { patients }\end{array}$} & \multicolumn{2}{|c|}{ Imprint cytology } & \multicolumn{2}{|c|}{ Frozen sectioning } & \multicolumn{2}{|c|}{ Immunohistochemistry } \\
\hline & & Sensitivity & Specificity & Sensitivity & Specificity & Sensitivity & Specificity \\
\hline Nagashima et a $\left.\right|^{31}$ & 124 & 62 & 99 & 79 & 100 & $\mathrm{~N} / \mathrm{A}$ & $N / A$ \\
\hline Sauer et $\mathrm{a}^{3^{33}}$ & 214 & 51 & 98 & 75 & 99 & $\mathrm{~N} / \mathrm{A}$ & $\mathrm{N} / \mathrm{A}$ \\
\hline Leidenius et $\mathrm{al}^{32}$ & 375 & 68 & 99 & 83 & 99 & $\mathrm{~N} / \mathrm{A}$ & $\mathrm{N} / \mathrm{A}$ \\
\hline Beach et $\mathrm{a}^{37}$ & 32 & 69 & 100 & 54 & 100 & $\mathrm{~N} / \mathrm{A}$ & $\mathrm{N} / \mathrm{A}$ \\
\hline Celebioglu et $\mathrm{a}^{22}$ & 100 & 51 & $\mathrm{~N} / \mathrm{A}$ & 73.5 & $\mathrm{~N} / \mathrm{A}$ & 75.5 & $\mathrm{~N} / \mathrm{A}$ \\
\hline Safai et $\mathrm{al}^{6}$ & 49 & 90 & 100 & 90 & 100 & 90 & 100 \\
\hline Francz et $\mathrm{al}^{23}$ & $\mathrm{~N} / \mathrm{A}$ & 69.4 & 97.8 & 68.5 & 98.9 & 68.5 & 98.9 \\
\hline Upender et $\mathrm{al}^{10}$ & 40 & 91.7 & 100 & 95.8 & 100 & 100 & 100 \\
\hline Mori et $\mathrm{al}^{25}$ & 183 & 47.1 & 88.2 & 88.2 & 98.3 & $\mathrm{~N} / \mathrm{A}$ & $\mathrm{N} / \mathrm{A}$ \\
\hline Lumachi et $\mathrm{al}^{24}$ & 126 & 70.3 & 91.9 & 75.7 & 100 & $\mathrm{~N} / \mathrm{A}$ & $N / A$ \\
\hline Krishnamurthy et al ${ }^{9}$ & 100 & 50 & 100 & 72 & 97.5 & 78 & 100 \\
\hline Our study & 60 & 90 & 100 & 80 & 100 & 100 & 100 \\
\hline
\end{tabular}

Abbreviation: N/A, not available. 
thick eosinophilic background and hyperplasia of small cells lacking adhesion is an important determinant for the presence of false-negative results. ${ }^{29}$ Therefore, in case of LC, intraoperative FS has been considered superior to IC. It is also essential to mention that in strong suspicion of LC based on previously preceded fine-needle aspiration (FNA) in the initial lesion, rapid analysis with either IC or FS should be always accompanied by IHC as associated cellular morphological characteristics remain controversial even in permanent stains. Evaluation of the FNA of the SLN section by the same cytopathologist dedicated to the diagnostic approach of the initial lesion remains an important parameter for the result accuracy. Another specific disadvantage of FS is that in case of a small-sized SLN the possibility of tissue destruction with parallel absence of material for final histological examination is apparent. Therefore, FS cannot be performed on occasion because of inadequate safety of protecting the permanent specimen. ${ }^{30-33}$ Moreover, LC cells are morphologically similar to lymphoid cells, thus making identification of metastases more challenging on both IC and definitive postoperative histology. ${ }^{34}$ In our survey, statistical analysis could not be performed among LC-affected patients, and further association with false-negative results has not been reported because of the small number of recorded cases.

Recently described intraoperative molecular methods for the detection of metastatic BC to SLN detect mRNA expression of the epithelial marker cytokeratin 19 (CK19). CK19 is normally absent in the lymph node, and its high level of expression is encountered in the majority of BC cells. The number of copies of mRNA for CK19 can be assessed with the one-step nucleic acid amplification test (OSNA). Sensitivity and specificity of the OSNA modification for the detection of metastases have been elucidated in a few surveys using different research protocols. The disadvantage of the OSNA method is homogenization of SLN and the associated lack of further possibility of histological evaluation. ${ }^{35}$ Thus, the same sample cannot be analyzed by both molecular OSNA analysis and subsequent postoperative histological examination. In addition, due to different parts of SLN used for analysis in each of the two methods, inconsistent results have been referred between the OSNA test and final biopsy. Relevant cost is also high in comparison with FS and IC alternative techniques.

In conclusion, IC appeared to be marginally more sensitive than FS in detecting SLN metastatic activity. In our prospective study, we did not face the problem reported in the literature, regarding the detection of micrometastases and
ITCs. Overall accuracy was 98.75\%. Diagnostic management was considered controversial in four cases presented with extensive sinus histiocytosis of the SLN and minimal reaction with cytokeratin AE1/AE3 and one case of LC atypical micrometastasis. With regard to the primary tumor characteristics, we conclude that initial lesion size and lymphovascular invasion play a pivotal role in metastatic involvement of the ASLN with the dimensions of metastasis bearing no correlation with tumor size. Furthermore, IC may be strongly indicated also in case of extranodal extension, in which a macrometastasis is almost always associated with the invasion of adjacent soft tissue. This morphological feature is very important in SLN of BC patients, also for the prognostic point of view. ${ }^{36}$ False-positive results that could lead to unnecessary ALND are very rare. The sensitivity of $90 \%$ of our research compares favorably with other reported studies of IC and FS. ${ }^{12,22,23}$ Therefore, IC is definitely an easy, quick, sensitive, and accurate method for the intraoperative assessment of SLN in BC patients.

\section{Disclosure}

The authors report no conflicts of interest in this work.

\section{References}

1. Gipponi M, Bassetti C, Canavese G, et al. Sentinel lymph node as a new marker for therapeutic planning in breast cancer patients. $J$ Surg Oncol. 2004;85(3):102-111.

2. Noguchi M. Current controversies concerning sentinel lymph node biopsy for breast cancer. Breast Cancer Res Treat. 2004;84(3):261-271.

3. Richards AD, Lakhani SR, James DT, Ung OA. Intraoperative imprint cytology for breast cancer sentinel nodes: is it worth it? ANZ J Surg. 2013;83(7-8):539-544.

4. Clarke D, Leung E, Chachlani N, et al. Intraoperative assessment of sentinel node using imprint cytology. World J Surg. 2010;34(1):55-61.

5. Tew K, Irwig L, Matthews A, Crowe P, Macaskill P. Meta-analysis of sentinel node imprint cytology in breast cancer. Br J Surg. 2005;92(9):1068-1080.

6. Safai A, Razeghi A, Monabati A, Azarpira N, Talei A. Comparing touch imprint cytology, frozen section analysis, and cytokeratin immunostaining for intraoperative evaluation of axillary sentinel lymph nodes in breast cancer. Indian J Pathol Microbiol. 2012;55(2):183-186.

7. Bishop JA, Sun J, Ajkay N, Sanders MA. Decline in frozen section diagnosis for axillary sentinel lymph nodes as a result of the American College of Surgeons Oncology Group Z0011 Trial. Arch Pathol Lab Med. 2016;140(8):830-835.

8. Wong J, Yong WS, Thike AA, et al. False negative rate for intraoperative sentinel lymph node frozen section in patients with breast cancer: a retrospective analysis of patients in a single Asian institution. J Clin Pathol. 2015;68(7):536-540.

9. Krishnamurthy S, Meric-Bernstam F, Lucci A, et al. A prospective study comparing touch imprint cytology, frozen section analysis, and rapid cytokeratin immunostain for intraoperative evaluation of axillary sentinel lymph nodes in breast cancer. Cancer. 2009;115(7):1555-1562.

10. Upender S, Mohan H, Handa U, Attri AK. Intraoperative evaluation of sentinel lymph nodes in breast carcinoma by imprint cytology, frozen section and rapid immunohistochemistry. Diagn Cytopathol. 2009;37(12):871-875. 
11. Motomura K, Nagumo S, Komoike Y, Koyama H, Inaji H. Accuracy of imprint cytology for intraoperative diagnosis of sentinel node metastases in breast cancer. Ann Surg. 2008;247(5):839-842.

12. Contractor K, Gohel M, Al-Salami E, et al. Intra-operative imprint cytology for assessing the sentinel node in breast cancer: results of its routine use over 8 years. Eur J Surg Oncol. 2009;35(1):16-20.

13. Barranger E, Antoine M, Grahek D, Callard P, Uzan S. Intraoperative imprint cytology of sentinel nodes in breast cancer. J Surg Oncol. 2004;86(3):128-133.

14. Howard-McNatt M, Geisinger KR, Stewart JH 4th, Shen P, Levine EA. Is intraoperative imprint cytology evaluation still feasible for the evaluation of sentinel lymph nodes for lobular carcinoma of the breast? Ann Surg Oncol. 2012;19(3):929-934.

15. Wang Y, Li L, Xu W, et al. High sensitivity of touch imprint cytology in young patients with invasive lobular carcinoma of the breast. Tumour Boil. 2014;35(6):5741-5746.

16. Salem AA, Douglas-Jones AG, Sweetland HM, Mansel RE. Intraoperative evaluation of axillary sentinel lymph nodes using touch imprint cytology and immunohistochemistry. Part II. Results. Eur J Surg Oncol. 2006;32(5):484-487.

17. Somasundaram SK, Chicken DW, Waddington WA, Bomanji J, Ell PJ, Keshtgar MR. Sentinel node imaging in breast cancer using superficial injections: technical details and observations. Eur J Surg Oncol. 2009;35(12):1250-1256.

18. Somasundaram SK, Chicken DW, Keshtgar MR. Detection of the sentinel lymph node in breast cancer. Br Med Bull. 2007;84:117-131.

19. Motomura K, Nagumo S, Komoike Y, Koyama H, Inaji H. Intraoperative imprint cytology for the diagnosis of sentinel node metastases in breast cancer. Breast Cancer. 2007;14(4):350-353.

20. Jeruss JS, Hunt KK, Xing Y, et al. Is intraoperative touch imprint cytology of sentinel lymph nodes in patients with breast cancer cost effective? Cancer. 2006;107(10):2328-2336.

21. Usman T, Rowlands DC, England DW. Rapid preoperative assessment of axillary lymph node status using imprint cytology. Breast. 1999;8(3):101-103.

22. Celebioglu F, Sylvan M, Perbeck L, Bergkvist L, Frisell J. Intraoperative sentinel lymph node examination by frozen section, immunohistochemistry and imprint cytology during breast surgery-a prospective study. Eur J Cancer. 2006;42(5):617-620.

23. Francz M, Egervari K, Szollosi Z. Intraoperative evaluation of sentinel lymph nodes in breast cancer: comparison of frozen sections, imprint cytology and immunocytochemistry. Cytopathology. 2011;22(1):36-42.

24. Lumachi F, Marino F, Zanella S, Chiara GB, Basso SM. Touch imprint cytology and frozen-section analysis for intraoperative evaluation of sentinel nodes in early breast cancer. Anticancer Res. 2012;32(8):3523-3526.
25. Mori M, Tada K, Ikenaga M, et al. Frozen section is superior to imprint cytology for the intra-operative assessment of sentinel lymph node metastasis in stage I breast cancer patients. World J Surg Oncol. 2006;4:26.

26. Jannink I, Fan M, Nagy S, Rayudu G, Dowlatshahi K. Serial sectioning of sentinel nodes in patients with breast cancer: a pilot study. Ann Surg Oncol. 1998;5(4):310-314.

27. Leers MP, Schoffelen RH, Hoop JG, et al. Multiparameter flow cytometry as a tool for the detection of micrometastatic tumour cells in the sentinel lymph node procedure of patients with breast cancer. $J$ Clin Pathol. 2002;55(5):359-366.

28. Treseler P. Pathologic examination of the sentinel lymph node: what is the best method? Breast J. 2006;12(5 Suppl 2):S143-S151.

29. Creager AJ, Geisinger KR, Perrier ND, et al. Intraoperative imprint cytologic evaluation of sentinel lymph nodes for lobular carcinoma of the breast. Ann Surg. 2004;239(1):61-66.

30. Pogacnik A, Klopcic U, Grazio-Frkovic S, Zgajnar J, Hocevar M, Vidergar-Kralj B. The reliability and accuracy of intra-operative imprint cytology of sentinel lymph nodes in breast cancer. Cytopathology. 2005;16(2):71-76.

31. Nagashima T, Suzuki M, Yagata $H$, et al. Intraoperative cytologic diagnosis of sentinel node metastases in breast cancer. Acta Cytol. 2003; 47(6):1028-1032.

32. Leidenius MH, Krogerus LA, Toivonen TS, Von Smitten KJ. The feasibility of intraoperative diagnosis of sentinel lymph node metastases in breast cancer. $J$ Surg Oncol. 2003;84(2):68-73.

33. Sauer T, Engh V, Holck AM, et al. Imprint cytology of sentinel lymph nodes in breast cancer. Experience with rapid, intraoperative diagnosis and primary screening by cytotechnologists. Acta Cytol. 2003;47(5):768-773.

34. Manfrin E, Remo A, Falsirollo F, et al. Intra-operative frozen section technique for breast cancer: end of an era. Pathologica. 2011;103(6): 325-330.

35. Szychta P, Westfal B, Maciejczyk R, et al. Intraoperative diagnosis of sentinel lymph node metastases in breast cancer treatment with onestep nucleic acid amplification assay (OSNA). Arch Med Sci. 2016; 12(6):1239-1246.

36. Nottegar A, Veronese N, Senthil M, et al. Extra-nodal extension of sentinel lymph node metastasis is a marker of poor prognosis in breast cancer patients: a systematic review and an exploratory meta-analysis. Eur J Surg Oncol. 2016;42(7):919-925.

37. Johnston EI, Beach RA, Waldrop SM, Lawson D, Cohen C. Rapid intraoperative immunohistochemical evaluation of sentinel lymph nodes for metastatic breast carcinoma. Appl Immunohistochem Mol Morpho. 2006;14(1):57-62.
Breast Cancer - Targets and Therapy

\section{Publish your work in this journal}

Breast Cancer - Targets and Therapy is an international, peerreviewed open access journal focusing on breast cancer research, identification of therapeutic targets and the optimal use of preventative and integrated treatment interventions to achieve improved outcomes, enhanced survival and quality of life for the cancer patient.

\section{Dovepress}

The manuscript management system is completely online and includes a very quick and fair peer-review system, which is all easy to use. Visit http://www.dovepress.com/testimonials.php to read real quotes from published authors. 